\title{
Research on On-Line Monitoring Device for Metal Oxide Arrester
}

\author{
Wu Di, Pan Jianqiao, Jin Chaohui, Hu Leijian \\ State Grid Zhejiang Pinghu Power Supply Limited Company, Zhejiang Pinghu, 314200
}

Keywords: Metal oxide arrester, online monitoring device, resistive current

\begin{abstract}
In view of the damage or explosion phenomenon of metal oxide surge arresters (MOA) in the current power system, which is easily affected by external factors, this paper will study the online monitoring device for metal oxide arresters. Throughout the research process, the author begins with the basic concepts of MOA and the research significance of online monitoring devices, and masters the design significance and basic theory of the device. On this basis, through the hardware design and software design two modules to build the entire metal oxide arrester online monitoring device. At the same time, considering the scientific and realistic nature of the metal oxide arrester online monitoring device, the author will also carry out simulation experiments on the device at the end of the article, in order to improve the safety of online monitoring of metal oxide arresters and ensure the power system is operating normally.
\end{abstract}

As the basic energy source in China, electric energy is related to the normal development of national life and industrial production. In this context, the operating state of the power system has become the focus of national attention. If the power system fails to operate normally during the operation, it will have a great impact on the residents of China. Among the many devices in the power system, metal oxide surge arresters (MOAs) are among the most important devices, which can greatly reduce the line faults caused by lightning strikes in power systems. However, as far as the current situation is concerned, MOA equipment does not have too strong safety during the operation of the power system, and is highly susceptible to thermal damage, transients, resonant overvoltage and other factors, resulting in damage or even explosion problems. It is a serious threat to the normal operation of the power system. It can be seen that in order to ensure the normal operation of the power system, it is imperative to improve the security of the MOA. Currently, the most common method for research on MOA safety is to conduct preventive trials on MOA. Although this method can prevent the failure of MOA to a certain extent, it is not suitable for long-term because it has the characteristics of long test period, high labor intensity, high maintenance cost and easy to produce false alarms. Based on this, this paper will study the online monitoring device for metal oxide arresters.

\section{The Basic Concept of MOA and the Research Significance of Online Monitoring Device}

MOA is one of the key equipments in many power systems, mainly for the protection of lightning overvoltage and operating overvoltage. The MOA itself has a high nonlinearity and is close to the insulation state during operation, which can effectively suppress overvoltage and protect other devices. Compared with other arresters, MOA has stronger protection and reliability, and has the advantages of simple structure and large flow capacity. It is the most commonly used lightning arrester in current power systems. However, MOA still has many problems in the operation of the power system, which is embodied in two points. First, the MOA does not have a series gap. When the leakage current flows from the arrester valve in the MOA, it will cause the valve to generate heat and accelerate the aging of the arrester valve, thus causing damage to the arrester. Second, the arrester valve in the MOA will also be affected by many factors such as poor sealing, poor performance of the insulating bracket, environmental pollution, etc., so that the MOA valve piece It is becoming more and more degraded, causing damage to the arrester. In serious cases, the arrester will also explode. It can be seen that MOA has a large potential safety hazard, which seriously threatens the operation of the power system, and focuses on various fields of value. 


\section{Design of MOA Online Monitoring Device}

After the above research on the basic concept of MOA and the significance of online monitoring device, this paper will design the MOA online monitoring device in detail, and combine the design ideas from the perspective of hardware and software.

\subsection{Overall structure of the hardware system}

The MOA online monitoring device hardware system designed in this paper is mainly composed of three modules, namely data acquisition and processing module, liquid crystal display module and bus module. The hardware system mainly acts on the collection and processing of the resistive leakage current and the transmission of the detection result, and realizes the online monitoring of the MOA by the power system administrator. The workflow of the hardware system mainly includes three major processes: First, the small current sensor in the hardware module will collect the MOA leakage current signal signal and convert it into a voltage signal; then, the converted voltage signal is filtered, sampled and analyzed; finally, the analyzed data is transmitted to the monitoring center server by means of the 485 bus, which is convenient for the management to observe and analyze. The overall structure of the specific hardware system is shown in Figure 1.

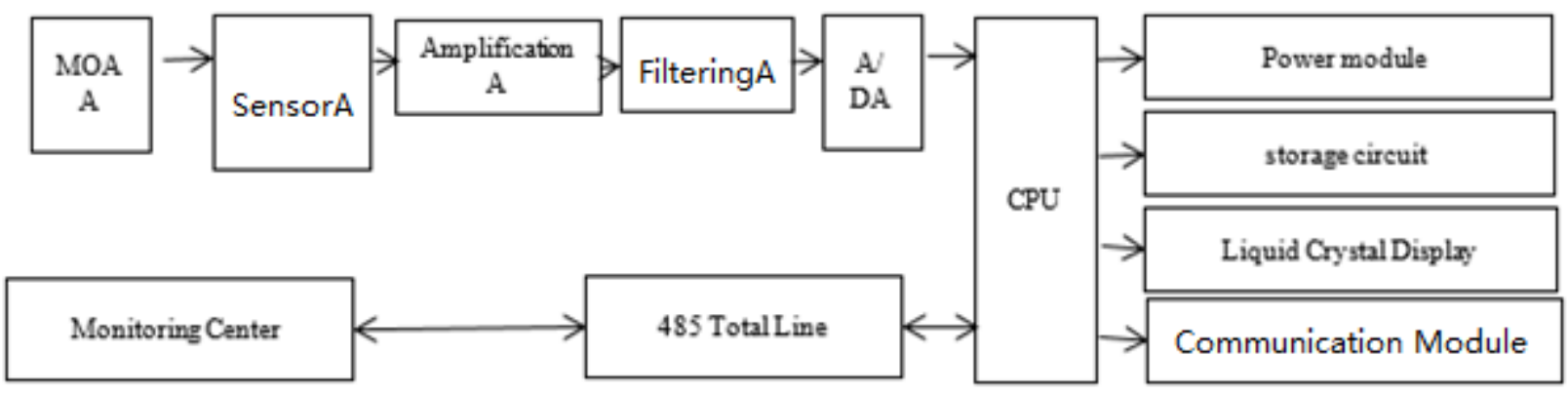

Figure 1 Hardware System Overall Structure

Sensor, amplification, filtering, power module, storage circuit, liquid crystal display, communication module fast, bus, monitoring center

\subsection{Leakage Current Acquisition Module Design}

The resistive current and capacitive current generated by MOA during operation are extremely small, usually only tens of microamps and hundreds of microamps. From this it can be concluded that the leakage current signal flowing in the MOA is also extremely small. At the same time, MOA's work environment is concentrated in substations with numerous power equipment, and there are many interference factors in this environment. Under this condition, considering the accuracy of leakage current acquisition, this paper will use a small current sensor (small CT) to collect the leakage current. In the actual application process, although the small CT will be affected by factors such as sensitivity and on-site environment, the sensor will not affect the primary wiring of the original high-voltage equipment, thus providing guarantee for the normal operation of the electrical equipment.

\subsection{Storage Unit Design}

Considering the rate of data sampling, this paper will temporarily store the sampled data in the external data memory after each sampling is completed, and then the data in the external data memory will be uniformly transmitted to the monitoring center after all the data is sampled. This reduces unnecessary time for each data transfer process. In the design process of the external data storage module, this paper will select the IS62C256 with the storage capacity of 32K as the external data storage chip, and latch the data with the latch 74HC573, so as to reduce the data pressure on the single-chip monitoring device. The schematic diagram of the specific data storage unit is shown in Figure 2. 


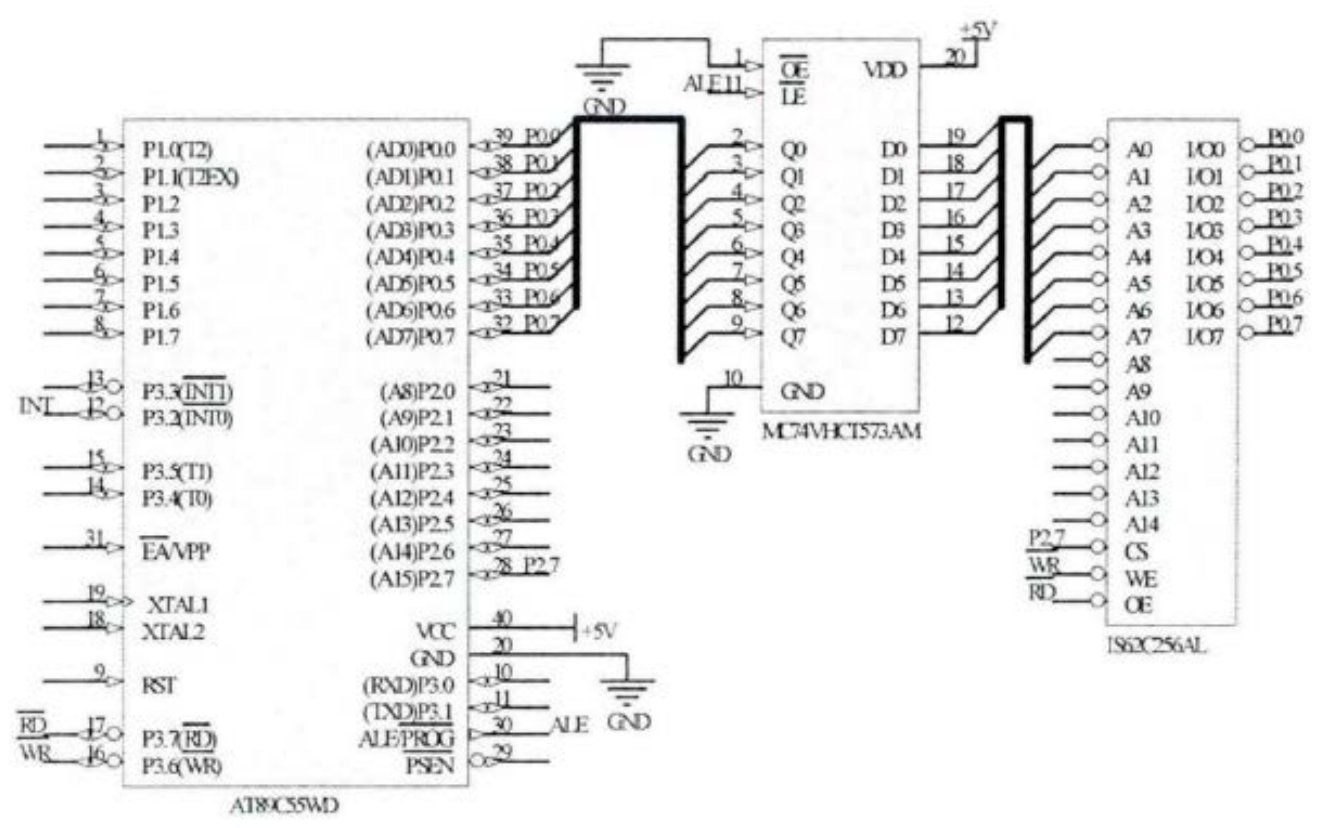

Figure 2 Data Storage Unit Schematic

\subsection{Display Unit Design}

The MOA online monitoring device mainly presents the leakage current of the MOA to the power system management personnel, so that the management personnel can analyze the MOA operating status in time. However, in order to visually present the leakage current in front of the manager, it is necessary to adopt a certain way to realize the display of the leakage current. At present, in the display field, the liquid crystal display has many advantages such as power saving, low radiation, and easy portability, and is the most widely used display at present. In the actual application process, the liquid crystal display mainly realizes the visualization of data by means of the physical properties of the liquid crystal. The liquid crystal module adopts LCD1602, which is based on the display content, and divides the LCD into three types: segment LCD, character LCD and dot matrix LCD. Among the three major LCD types, the character LCD has many features such as low cost, rich display and convenient use, and is the perfect substitute for the LED digital tube in the current display field. Based on this, this paper will use the character LCD to design the display unit of the MOA online monitoring device. The schematic diagram of the specific liquid crystal display circuit is shown in Figure 3.
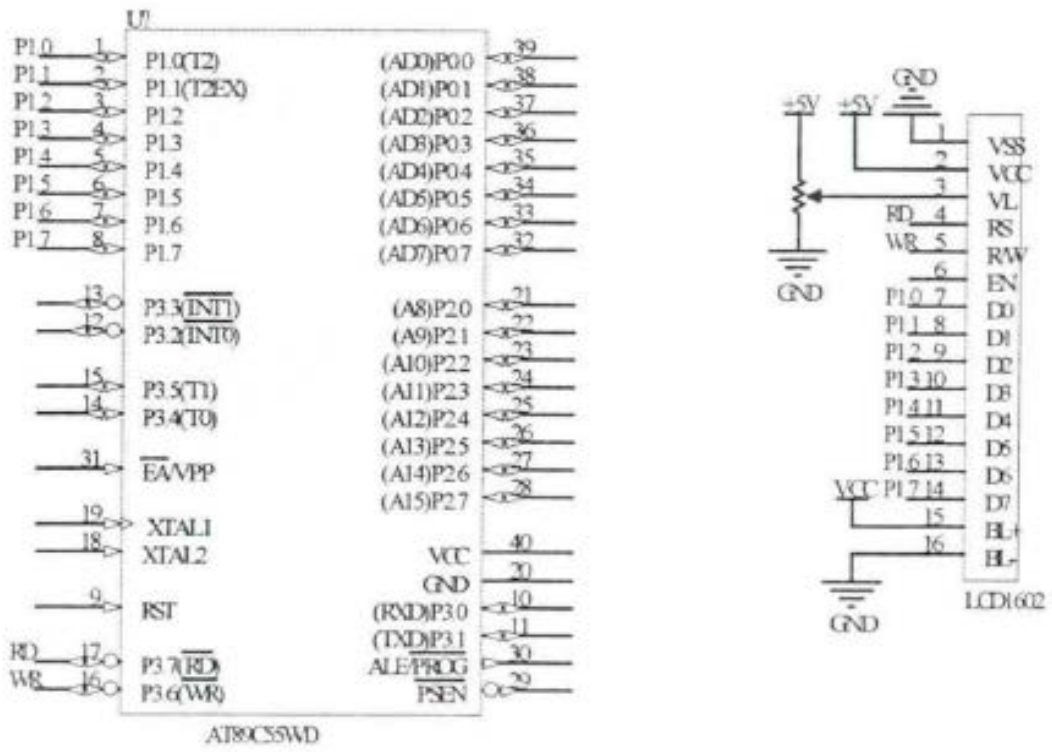

Figure 3 Schematic Diagram of the Liquid Crystal Display Circuit 


\subsection{Communication Unit Design}

Because the distance between the front-end data acquisition system of the MOA online monitoring device and the back-end monitoring center is relatively long, the communication process of the device lacks real-time performance. In order to make the front and rear modules in the MOA online monitoring device transmit long-distance data at a faster speed, this paper will use the RS485 communication bus to design the communication unit of the online monitoring device. The characteristics of the RS485 communication bus are as follows:

(1) The maximum communication distance and maximum transmission rate in the RS485 communication bus are $1200 \mathrm{~m}$ and $10 \mathrm{Mbps}$, respectively. Assuming that the RS485 communication bus needs to transmit a longer distance, a 485 communication repeater needs to be added to the RS485 communication bus to realize long-distance data transmission;

(2) RS485 communication bus interface has better anti-interference. Since the RS485 communication bus has a combination of a balanced driver and a differential receiver, it is more resistant to common mode interference when it intersects with other communication buses;

(3) Since the RS485 communication bus only specifies the electrical characteristics of the receiving end and the transmitting end, the data protocol is not specified accordingly. Therefore, when designing the RS485 communication bus, it is also necessary to separately design the protocol of the communication scheme based on the physical transmission requirements of the RS485 communication bus;

(4) In the communication mode, the RS485 communication bus can adopt the communication method of two-wire system and half-duplex communication, and can also use the four-wire system and full-duplex communication mode to support data transmission and reception simultaneously. The schematic diagram of the specific communication unit is shown in Figure 4.

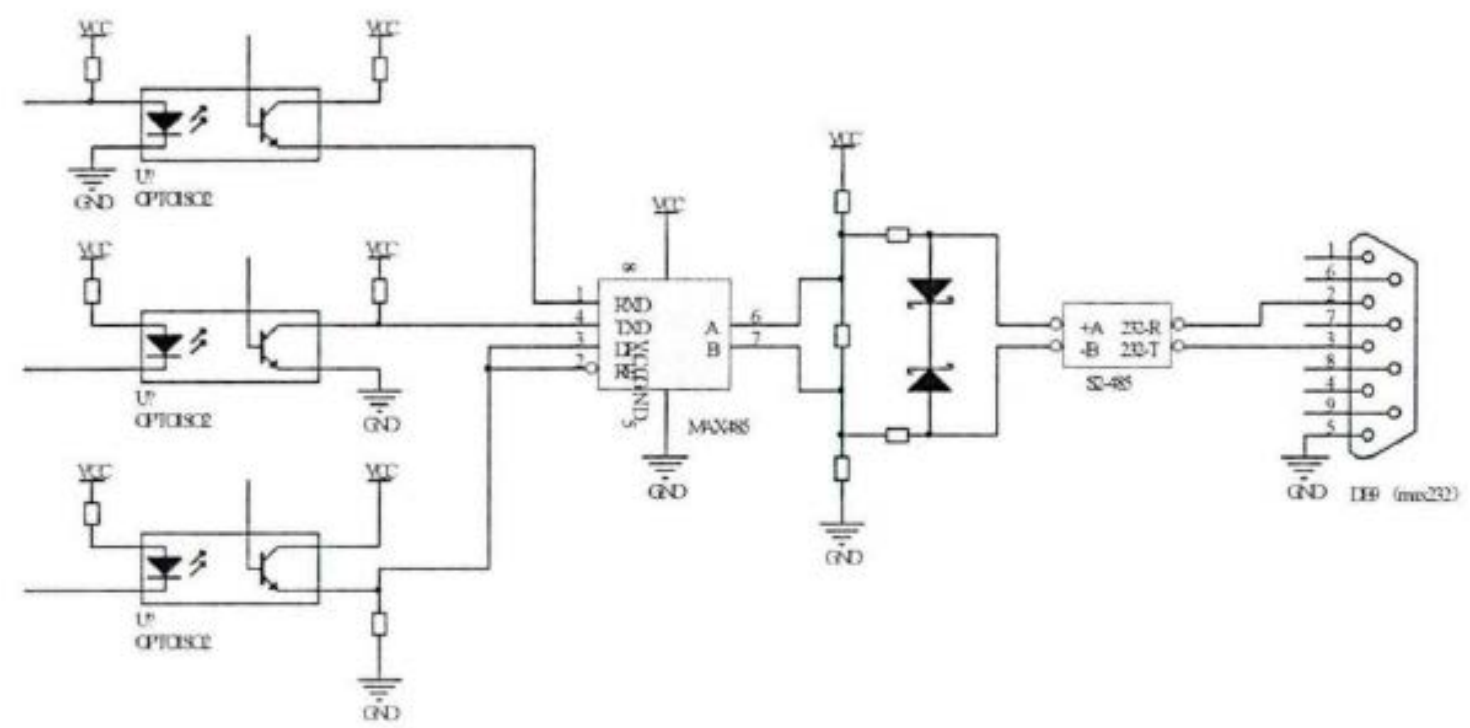

Figure 4 Communication Unit Schematic

Because the MOA online monitoring device has different levels of the MCU and the computer, the former level format is TTL level, and the latter format is RS232, which makes direct communication between the MCU and the computer, and the basic conversion demand must be realized by means of the level shifting device. Based on this, this paper first uses MAX485 to convert the TTL level format of the single-chip microcomputer and then transmits it for long distance. Then, it uses S2-485 to convert the RS485 interface signal to make it RS232 signal. Finally, the nine-pin interface DB9 converts the RS232 signal to make the RS232 signal become a serial port signal, enabling direct communication between the microcontroller and the computer. At the same time, considering the interference in the communication process, this paper will introduce optocouplers between the MCU and MAX485 for isolation. 


\section{Software Part Design}

After designing the hardware part of the MOA online monitoring device, it is necessary to design its software department program to build a complete online monitoring device. The specific software part is designed as follows:

\subsection{System Data Acquisition Program Design}

The specific system data acquisition program design is shown in Figure 5.

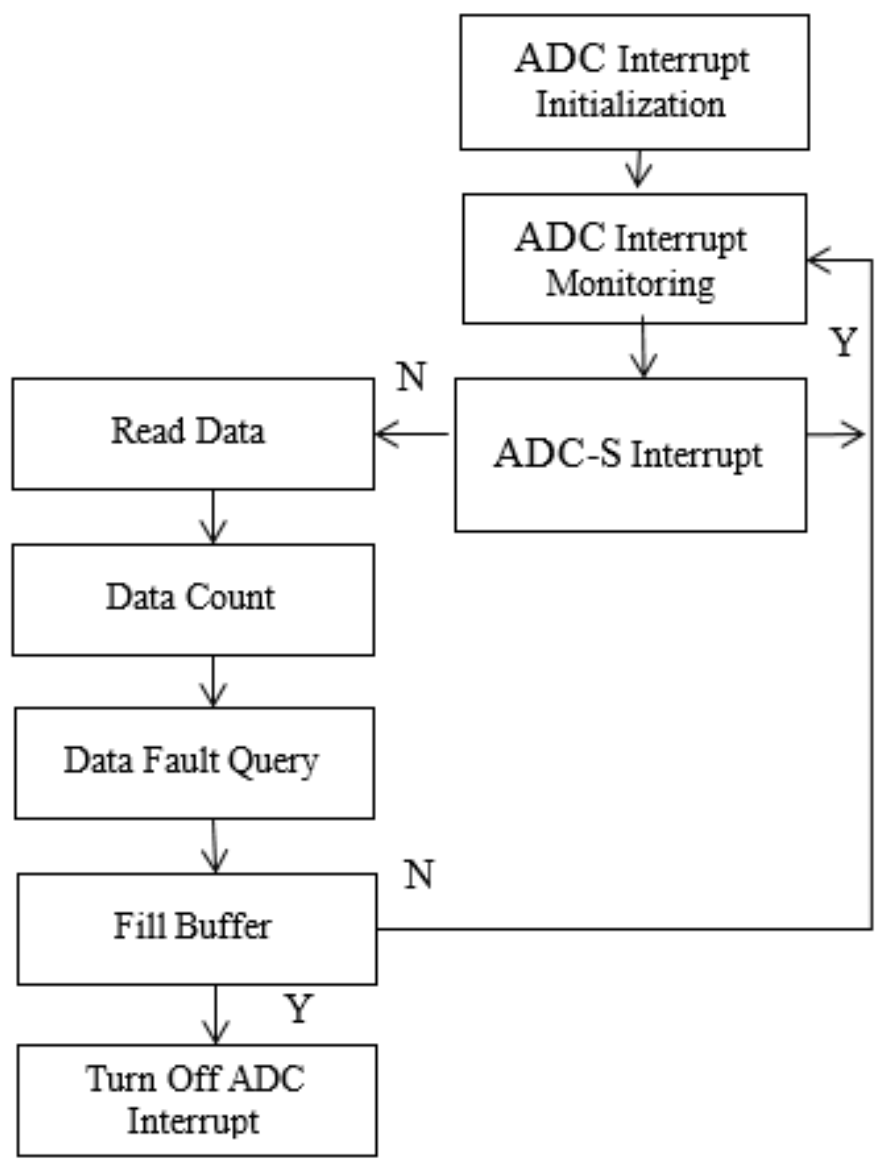

Figure 5 System Data Acquisition Program Design

As shown in the figure above, after the MOA online monitoring device is started, the initialization of the signal acquisition department will be completed by the system main function, and the signal acquisition function will be started at the same time. In the system data acquisition program, data collection and storage are mainly realized by recording interrupts.

And in this way, the number of data collection is counted. Introducing the interrupt service function in the data acquisition module not only improves the real-time and synchronization of the data acquisition process, but also further reduces the error of the MOA online monitoring device.

\subsection{Communication System Programming}

In the communication unit of the MOA online monitoring device, after the hardware program configuration initialization is performed, the main program writes the communication data using the data transfer buffer register. The specific communication system program design is shown in Figure 6. 


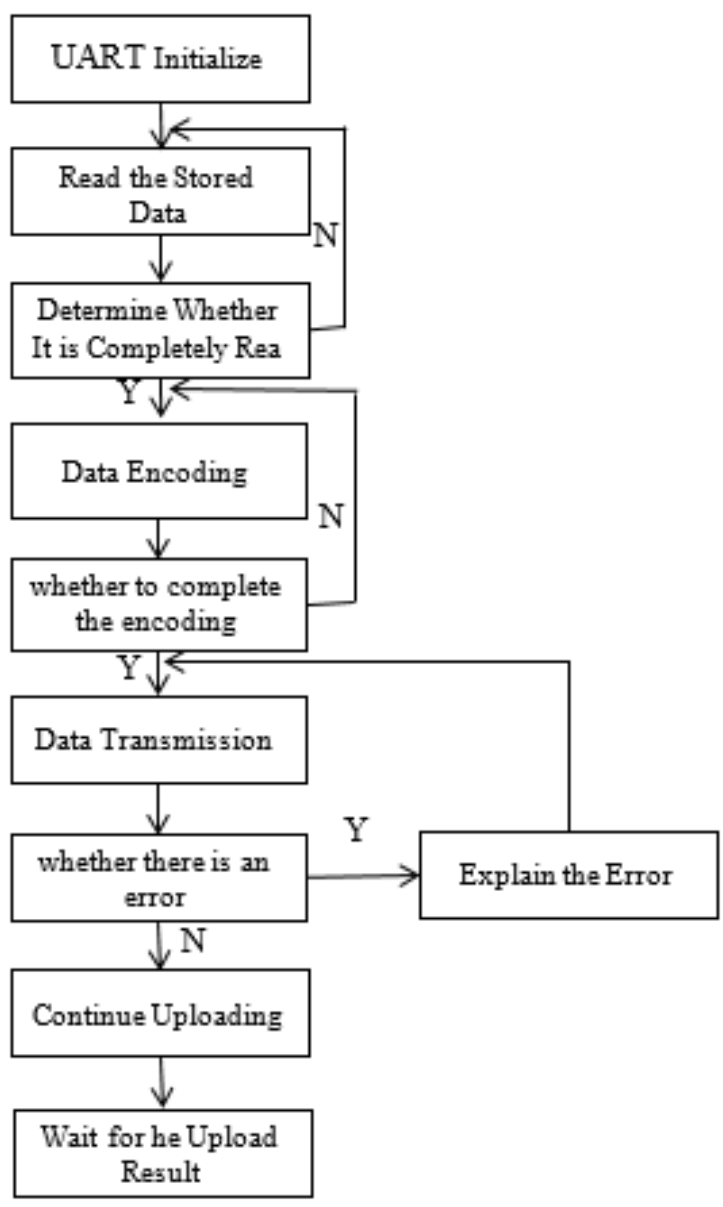

Figure 6 Communication System Programming

\section{Simulation Experiment}

In order to verify whether the MOA online monitoring device designed in the above is scientific and realistic, this paper will simulate the effect of extracting resistive current in the online monitoring device under the condition of MOA degradation. In this simulation experiment, the simulation model of the three-phase MOA equivalent capacitance is selected to conduct experiments, and the peak value of the resistive current under the normal condition of MOA is compared with the resistive current in the case of MOA degradation. The specific experimental results are shown in Table 1.

Table 1 Experimental Results

\begin{tabular}{lll}
\hline Different & $\begin{array}{l}\text { The Peak Value of the Resistive Current } \\
\text { under Normal Conditions of MOA }\end{array}$ & $\begin{array}{l}\text { The Peak Value of Resistive Current in } \\
\text { the Case of MOA Degradation }\end{array}$ \\
\hline A & 1.793 & 3.912 \\
B & 3.167 & 5.205 \\
C & 3.173 & 5.288
\end{tabular}

As shown above, in the case of MOA degradation, the peak value of the resistive current extracted by the MOA online monitoring device designed in this paper is significantly higher than the peak value of the resistive current under the normal condition of MOA. This phenomenon indicates that the device can effectively extract the MOA degradation. The peak value of the resistive current provides a clear basis for the power system management personnel to judge the MOA degradation, so that it can grasp the MOA operating status in time. 


\section{Conclusion}

In summary, MOA is one of the important equipments of the entire power system, and the performance of the equipment will directly affect the operating state of the power system. In this paper, based on the current lack of reasonable monitoring methods in MOA monitoring, the overall structure of hardware system, leakage current acquisition module design, storage unit design and display are based on the basic concepts of MOA and the significance of online monitoring devices. Unit design, system data acquisition program design with software module, communication system program design constitute MOA online monitoring device, and the scientific and practical value of the device is endowed by the final simulation experiment. In this way, MOA security is improved and a strong guarantee for the safe operation of the power system is provided.

\section{References}

[1] Dongyi Zhao, Shuhui Hu, Lanyi Wang, Jiajun Wang. Research and Development of On-Line Monitoring Technology for Metal Oxide Arresters for Intelligent Substations[J]. Electric Porcelain Arrestors, 2013(05): 66-72.

[2] Min Lei, Quan Wang, Jiliang Fu, Wei Wang, Jun Zhang, Xiang Liu, Gaoning Nie. Research on On-Site Calibration Technology of Metal Oxide Arrester Monitoring Device[J]. High Voltage Apparatus, 2017, 53(12): 93-98 +105.

[3] Caijian Luo. Research on Online Monitoring Algorithm of Metal Oxide Arrester Considering Harmonic Voltage Influence[J]. Electric Porcelain Arrestors, 2016(03): 53-57.

[4] Changjie Chen, Sun Jin, Cao Hui. Fault Handling of Metal Oxide Arrester Online Monitor[J]. Electric World, 2015, 56(02): 26.

[5] Zhongjiang Yang, Hongliang Cao, Pengfei Li, Zehuang Chen, Jiang Zhu. On-line Monitoring of Metal Oxide Arresters Based on Genetic Algorithm[J]. High Voltage Engineering, 2015, 41(09): 3104-3109.

[6] Guixian He, Hongyan Xing, Xinyuan Ji, Wei Xu. Harmonic Correction and Research on On-line Monitoring of Metal Oxide Arresters[J]. Journal of Electronic Measurement and Instrument, 2017, 31(10): 1549-1554. 